\title{
Rationals on long closed horocycle flows
}

\author{
Emek Demirci Akarsu
}

Department of Mathematics, Recep Tayip Erdogan University, Rize, Turkey

Received: 3 February 2020, Accepted: 25 February 2020

Published online: 18 March 2020.

\begin{abstract}
In this paper, we investigate the distribution theorem of rational points on the closed horocycles. Sarnak proved that the long closed horocycles are equidistributed on the modular surface. We embed rational points on such horocyles on the modular surface and its tangent bundle, and study their distribution problem.
\end{abstract}

Keywords: Long closed horocycles, distribution, Kloosterman sums.

\section{Introduction}

In homogeneous dynamics one can take a finite Lie group $G$ and a lattice $\Gamma$ (a lattice $\Gamma$ in $G$ is a discrete subgroup such that there is a fundamental domain $\mathscr{F}_{\Gamma}$ of the left $\Gamma$-action on $G$ with finite left Haar measure), and consider the natural actions of subgroup $H$ of $G$ on the quotient $\Gamma \backslash G$. The quotient $\Gamma \backslash G$ is a homogeneous space of the form $\{\Gamma g: g \in G\}$ and $H$ acts by right multiplication.

Let $\mathbb{H}$ be the upper half-plane and $\Gamma=\operatorname{SL}(2, \mathbb{Z})$ be the modular group and $\mathscr{M}:=\Gamma \backslash \mathbb{H}$ be the modular surface. The closed horocycles of length $1 / y$ become equidistributed on the unit tangent bundle $T^{1} \mathscr{M}$ of the hyperbolic surface $\mathscr{M}$ of finite area, as $y \rightarrow 0$ [8]. That is, for any $f:[0,1] \times \mathscr{M} \rightarrow \mathbb{R}$ bounded continuous function we have

$$
\lim _{y \rightarrow 0} \int_{0}^{1} f(x, x+\mathrm{i} y) d x=\int_{0}^{1} \int_{\mathscr{M}} f(\xi, x+\mathrm{i} y) d \xi \frac{d x d y}{y^{2}} .
$$

We have in particular $T^{1} \mathscr{M}=\Gamma \backslash T^{1} \mathbb{H}$. The asymptotic behavior of averages along closed horocycles in finite volume has been investigated by the several authors, see Zagier [10], Sarnak [8], Eskin and McMullen [4], Hejhal [7] and Strömbergsson [9]. Their approaches vary from exploit mixing to harmonic analysis and to Ratner's classification of measures invariant under the unipotent flows.

Let $\Gamma \backslash \mathbb{H}$ be non-compact, that is, it has at least one cusp. For any $y>0$, the curve $\{(p / q+i y, 0) \mid p / q \in \mathbb{Q}\}$ is an example of an orbit of the horocyclic flow on $T^{1} \mathbb{H}$. See section 2 for details.

We denote $\mathbb{Z}_{q}^{\times}$by the multiplicative group of integers modulo $q$. Our investigation is concerned with the equidistribution of the point set

$$
\Gamma\left\{(p / q+i y, 0) \mid p \in \mathbb{Z}_{q}^{\times}\right\}
$$

as $q \rightarrow \infty$ and $y \rightarrow 0$. We embed rational points on closed horocycles on the modular surface $\Gamma \backslash \mathbb{H}$ and later its unit tangent bundle. To do this we exploit Sarnak's equidistribution theorem on the modular surface [8]. 
Our analytic way of handling equidistribution questions is to use bounds for Fourier coefficients. We will seek whether the rational points on horocycle flows are equidistributed in a suitable measure theoretical sense. We addressed this issue by exploiting Weil's bounds on Kloosterman sums.

Assume $f: \mathbb{R} \times \mathbb{H} \rightarrow \mathbb{R}$ is given. We also assume $f$ is periodic in the first variable, i.e. $f(\xi+1, z)=f(\xi, z)$, and modular with respect to the second variable. That is, $f(\xi, \gamma z)=f(\xi, z)$ for all $\gamma \in \Gamma$. Note that this yields

$$
f(\xi, z+1)=f(\xi, z)
$$

since

$$
\left(\begin{array}{ll}
1 & 1 \\
0 & 1
\end{array}\right) \in \Gamma
$$

This means that $f$ is periodic, and we can expand $f$ in a Fourier series

$$
f(\xi, x+\mathrm{i} y)=\sum_{m, n \in \mathbb{Z}} \hat{f}_{m, n}(y) e(m \xi+n x),
$$

where $e(t):=\mathrm{e}^{2 \pi \mathrm{i} t}$. The Fourier coefficients are given by

$$
\hat{f}_{m, n}(y)=\int_{0}^{1} \int_{0}^{1} f(\xi, x+\mathrm{i} y) e(-m \xi-n x) d x d y
$$

We can identify $f$ naturally as a function on $\mathbb{T} \times \mathscr{M}$. Let

$$
K_{\sigma}(f):=\sum_{(m, n) \neq(0,0)}\left|\hat{f}_{m, n}\left(\frac{1}{\sigma}\right)\right| \max \{|m|,|n|\}^{1 / 2}
$$

and define the space of functions $f$ with finite $K_{\sigma}(f)$ as

$$
\mathscr{K}_{\sigma}=\left\{f: \mathbb{T} \times \mathscr{M} \rightarrow \mathbb{C} \mid K_{\sigma}(f)<\infty\right\}
$$

We denote Euler's totient function by $\varphi(q)$, that is

$$
\varphi(q)=\#\{n \leq q, \operatorname{gcd}(n, q)=1\}=\# \mathbb{Z}_{q}^{\times} .
$$

Theorem 1. Given $\varepsilon>0$, there is a constant $C_{\varepsilon}>0$ such that for all $q \in \mathbb{N}, \sigma>0$ and $f \in \mathscr{K}_{\sigma}$, we have

$$
\left|\frac{1}{\phi(q)} \sum_{p \in \mathbb{Z}_{q}^{\times}} f\left(\frac{p}{q}, \frac{p}{q}+\mathrm{i} \frac{\sigma}{q^{2}}\right)-\int_{\mathbb{T}^{2}} f\left(\xi, x+\mathrm{i} \frac{1}{\sigma}\right) d \xi d x\right| \leq C_{\varepsilon} K_{\sigma}(f) q^{-1 / 2+\varepsilon} .
$$

The proof of theorem is contained in Section 3. The proof follows from the classical bounds on Kloosterman sums. We later extend the result of Theorem 1 to the unit tangent bundle of the modular surface $\Gamma \backslash \operatorname{SL}(2, \mathbb{R})$, namely Theorem 2 below.

As a higher dimensional variant of Equation (10) of the distribution of rational points on horospheres has been investigated in [3]. However their ergodic way of proof differs from our number theoretical proof. It is interesting to note that their ergodic-theoretic proof only works in the higher dimensional setting. Limit theorems for rational points on the metaplectic horocycle and their applications to the incomplete Gauss sums are investigated in [2]. 
We will now extend Theorem 1 , replacing $\mathbb{H}$ by $G:=\operatorname{SL}(2, \mathbb{R})$. We use notation $\mathscr{M}_{1}$ stands for $\Gamma \backslash G$. We now consider the distribution of the point set

$$
\Gamma\left\{(p / q+i y, 0) \mid p \in \mathbb{Z}_{q}^{\times}\right\}
$$

as $q \rightarrow \infty$ and $\sigma$ fixed and $y \rightarrow 0$ in the unit tangent bundle $\mathbb{T}^{1} \mathscr{M}_{1}$. We define $f: \mathbb{T} \times \mathscr{M}_{1} \rightarrow \mathbb{C}$. Let

$$
N_{\sigma}(f):=\sum_{(m, n) \neq(0,0)}\left|\hat{f}_{m, n}\left(\frac{1}{\sigma}, \frac{\pi}{2}\right)\right| \max \{|m|,|n|\}^{1 / 2}
$$

where $\hat{f}_{m, n}(y, \phi)=\int_{0}^{1} \int_{0}^{1} f(\xi, x+\mathrm{i} y, \phi) e(-m \xi-n x) d \xi d x$ is Fourier coefficient of $f$ with $y=\frac{1}{\sigma}$ and $\phi=\frac{\pi}{2}$ and

$$
\mathscr{N}_{\sigma}=\left\{f: \mathbb{T} \times \mathscr{M}_{1} \rightarrow \mathbb{C} \mid N_{\sigma}(f)<\infty\right\} .
$$

As mentioned earlier, $f$ is periodic, which means that $f(\xi+1, z, \phi)=f(\xi, z, \phi)$, and $f\left(\xi, \gamma z, \phi_{\gamma}\right)=f(\xi, z, \phi)$ with $\gamma z=g z$ and $\phi_{\gamma}=\phi+\arg (c z+d)$ for all $\gamma \in \Gamma$ and $g \in G$.

Theorem 2. For all $q \in \mathbb{N}, \sigma>0$ and $f \in \mathscr{N}_{\sigma}$ such that $f(\xi, z, \phi)=f(\xi, M)$ with $M:=(z, \phi) \in G$, we have

$$
\left|\frac{1}{\varphi(q)} \sum_{p \in \mathbb{Z}_{q}^{\times}} f\left(\frac{p}{q}, \frac{p}{q}+\mathrm{i} \frac{\sigma}{q^{2}}, 0\right)-\int_{\mathbb{T}^{2}} f\left(\xi, x+\mathrm{i} \frac{1}{\sigma}, \frac{\pi}{2}\right) d \xi d x\right| \leq C_{\varepsilon} N_{\sigma}(f) q^{-1 / 2+\varepsilon} .
$$

Remark.In Theorem 1 and 2, we have studied the limit distribution of rationals on horocycle flows with $\sigma$ fixed.

\section{Preliminaries}

We use the common notation for $z=x+\mathrm{i} y \in \mathbb{C}$, with the real part $\operatorname{Re} z=x$ and the imaginary part $\operatorname{Im} z=y$. Let us take matrix $g=\left(\begin{array}{ll}a & b \\ c & d\end{array}\right)$ with determinant 1, i.e. $a d-b c=1$. This forms a group under matrix multiplication. We call this group the unimodular group and denote it by $G:=\operatorname{SL}(2, \mathbb{R})$.

Möbious transformation is a map $\mathfrak{M}(\mathbb{H}):=\hat{\mathbb{C}} \rightarrow \widehat{\mathbb{C}}$ acting on the Riemann sphere $\widehat{\mathbb{C}}=\mathbb{C} \cup\{\infty\}$ by fractional linear transformations

$$
z \rightarrow g z=\frac{a z+b}{c z+d}
$$

where $z \in \mathbb{C}$. Notice that $-g \equiv g$.

The complex plane splits into three $\operatorname{SL}(2, \mathbb{R})$-invariant subspaces under the action (14), the upper half-plane, the real line $\hat{\mathbb{R}}$ and the lower upper half-plane. We focus on the upper half-plane all throughout. The complex upper half-plane $\mathbb{H}$ is defined as

$$
\mathbb{H}=\{z \in \mathbb{C}: \operatorname{Im} z>0\} .
$$

A generalized circle is a circle or a straight line in $\widehat{\mathbb{C}}$. A horocycle is a horizontal line or a circle joining $\hat{\mathbb{R}}$ tangentially. It is important to note that $\mathfrak{M}(\mathbb{H})$ takes horocycles to horocycles.

The main objects of the hyperbolic plane are the straight lines and semicircles orthogonal to the real axis, both defined in terms of Euclidean objects in $\mathbb{C}$. The upper half-plane $\mathbb{H}$ is equipped with the metric

$$
d s^{2}=\frac{d x^{2}+d y^{2}}{y^{2}}
$$


We can find the shortest curve between given any two points of $\mathbb{H}$ with respect to the metric above, in fact the distance between these points is measured along with the unique geodesic. Let $\rho:[a, b] \rightarrow \mathbb{H}$ be piecewise $C^{1}$ path such that $\rho=\{z(t)=x(t)+\mathrm{i} y(t) \in \mathbb{H} \mid t \in[0,1]\}$. We then define hyperbolic length of $\rho$

$$
\operatorname{length}(\rho)=\int_{\rho} \frac{|d z|}{\operatorname{Im} z}=\int_{a}^{b} \frac{|d z / d t| d t}{y(t)} .
$$

We denote hyperbolic distance $d_{\mathbb{H}}(z, w)$ by the shortest length of length $(\rho)$ for each pair of points $z, w \in \mathbb{H}$. Moreover $\left(\mathbb{H}, d_{\mathbb{H}}\right)$ is a metric space.

Let $A \subset \mathbb{H}$. Then the hyperbolic area of the set $A$ is given by the integral

$$
\operatorname{area}_{\mathbb{H}}(A)=\int_{A} \frac{d x d y}{y^{2}}
$$

The hyperbolic length and area are invariant under the action of Möbious transformation $\mathfrak{M}(\mathbb{H})$.

Define the discrete subgroup of $G$, the modular group

$$
\mathrm{SL}(2, \mathbb{Z})=\left\{\left(\begin{array}{ll}
a & b \\
c & d
\end{array}\right): a, b, c, d \in \mathbb{Z}, a d-b c=1\right\} .
$$

The generators of this group are the elements $\left(\begin{array}{ll}1 & 1 \\ 0 & 1\end{array}\right),\left(\begin{array}{ll}0 & -1 \\ 1 & 0\end{array}\right)$. The Dirichlet fundamental domain, which is a subset of a given topological space which contains exactly one point from each of the orbits of the group action on that space, of $\operatorname{SL}(2, \mathbb{Z})$ in $\mathbb{H}$ is

$$
\mathscr{F}_{\Gamma}=\{z \in \mathbb{H}:-1 / 2 \leq x \leq 0,|z| \geq 1\} \cup\{z \in \mathbb{H}: 0<x<1 / 2,|z|>1\} .
$$

We define the unit tangent bundle $T^{1} \mathbb{H}$ of the hyperbolic plane $\mathbb{H}$ by $(z, \theta)$, where $z$ is a complex coordinate in $\mathbb{H}$ and $\theta \in \mathbb{R} \backslash 2 \pi \mathbb{Z}$ is the angular variable measuring the orientation of an arrow attached to the complex coordinate $z$. We can simply identify $T^{1} \mathbb{H}$ with $\mathbb{H} \times(\mathbb{R} / 2 \pi \mathbb{Z})$. The definition of an action of the projective special linear group $\operatorname{PSL}(2, \mathbb{R})$ on $T^{1} \mathbb{H}$ is then given by

$$
g:(z, \theta) \rightarrow\left(g z, \theta-2 \beta_{g} z\right)
$$

where $g z$ corresponds the usual action of fractional linear transformation and the transformation of the angle $\theta$ comes from the transformation property of a tangent vector $d z$ under $g \in G, \beta_{g} z=\arg (c z+d)$. Left multiplication also becomes an action of $G$ on $\mathbb{H} \times[0,2 \pi)$ that allows us to write

$$
\left(\begin{array}{ll}
a & b \\
c & d
\end{array}\right)(z, \phi)=\left(\frac{a z+b}{c z+d}, \phi+\arg (c z+d)\right),
$$

where $\left(\begin{array}{ll}a & b \\ c & d\end{array}\right) \in \Gamma$. The positive horocycle flow and the negative horocycle flow on the unit tangent bundle $T^{1} \mathscr{M}_{1} \simeq \Gamma \backslash \operatorname{PSL}(2, \mathbb{R})$ is given

$$
\operatorname{PSL}(2, \mathbb{R}) \rightarrow \operatorname{PSL}(2, \mathbb{R}), \quad g \mapsto g\left(\begin{array}{ll}
1 & t \\
0 & 1
\end{array}\right)
$$

and

$$
\operatorname{PSL}(2, \mathbb{R}) \rightarrow \operatorname{PSL}(2, \mathbb{R}), \quad g \mapsto g\left(\begin{array}{ll}
1 & 0 \\
t & 1
\end{array}\right),
$$

respectively. For more details about homogeneous dynamics we refer Bekka and Mayer's book [1]. 


\section{Proof of Theorem 1}

We will need following lemma in the proof of the theorem. Let us first define Kloosterman sum

$$
K(m, n ; q)=\sum_{p \in \mathbb{Z}_{q}^{\times}} e\left(\frac{m p+n \bar{p}}{q}\right)
$$

for integers $m, n$ and $q \geq 1$, where $\bar{p}$ denotes the multiplicative inverse of $p \bmod q$.

Lemma 1. Given $\varepsilon>0$ and for all $m, n, q \in \mathbb{N}$, there is a constant $C_{\varepsilon}>0$ such that

$$
\frac{1}{\varphi(q)}|K(m, n ; q)| \leq C_{\varepsilon} q^{-1 / 2+\varepsilon} \operatorname{gcd}(m, n, q)^{1 / 2} .
$$

Proof. The bound given in Lemma 1 is a direct consequence of the Weil bound for Kloosterman sums [5],

$$
|K(m, n ; q)| \leq \tau(q) \operatorname{gcd}(m, n, q)^{\frac{1}{2}} \sqrt{q},
$$

where $\tau(q)$ is the number of positive divisors of $q$. It is well known that upper bound for $\tau(q)$ [6, Chapter XVI] is $c_{\varepsilon} q^{\varepsilon}$, for any $\varepsilon>0$. By using standard bound for $\varphi(q) \gg_{\varepsilon} q^{1-\varepsilon}$ for any $\varepsilon>0$ and Weil's bound on Kloosterman sum (25), we have

$$
\frac{1}{\varphi(q)}|K(m, n ; q)| \leq \frac{C_{\varepsilon} q^{\varepsilon} \operatorname{gcd}(m, n, q)^{1 / 2} q^{1 / 2}}{q^{1-\varepsilon}}
$$

for any $\varepsilon>0$.

We are now ready to supply the proof of Theorem 1.

Proof. Integers $p$ and $q$ are given, we claim that there is $\gamma=\left(\begin{array}{ll}a & b \\ c & d\end{array}\right) \in \Gamma$, such that

$$
\left(\begin{array}{ll}
a & b \\
c & d
\end{array}\right)\left(\frac{p}{q}+\mathrm{i} \frac{\sigma}{q^{2}}\right)=\left(-\frac{\bar{p}}{q}+\mathrm{i} \frac{1}{\sigma}\right)
$$

where $p \bar{p} \equiv 1 \bmod q$. To prove this, notice that

$$
\left(\begin{array}{ll}
a & b \\
c & d
\end{array}\right)\left(\frac{p}{q}+\mathrm{i} \frac{\sigma}{q^{2}}\right)=\left(\frac{a\left(\frac{p}{q}+\mathrm{i} \frac{\sigma}{q^{2}}\right)+b}{c\left(\frac{p}{q}+\mathrm{i} \frac{\sigma}{q^{2}}\right)+d}\right)
$$

by fractional linear transformations. By choosing $c=q$ and $d=-p$, we reduce the left hand side of (30) to

$$
(\underbrace{\frac{\left(-\frac{a d}{c}+\mathrm{i} \frac{a \sigma}{c^{2}}\right)+b}{c \mathrm{i}\left(\frac{\sigma}{c^{2}}\right)}}_{\frac{a}{c}+\mathrm{i} \frac{1}{\sigma}}) .
$$

Since $a d=1+b c, a p \equiv-1 \bmod q$ and $a=-\bar{p}$. Because $f$ is invariant under $\Gamma$, we simply have

$$
\frac{1}{\varphi(q)} \sum_{p \in \mathbb{Z}_{q}^{\times}} f\left(\frac{p}{q}, \frac{p}{q}+\mathrm{i} \frac{\sigma}{q^{2}}\right)=\frac{1}{\varphi(q)} \sum_{p \in \mathbb{Z}_{q}^{\times}} f\left(\frac{p}{q},-\frac{\bar{p}}{q}+\mathrm{i} \frac{1}{\sigma}\right) .
$$


We now use the Fourier expansion, then the left hand side of above equation equals

$$
\frac{1}{\varphi(q)} \sum_{p \in \mathbb{Z}_{q}^{\times}} \sum_{m, n \in \mathbb{Z}} \hat{f}_{m, n}\left(\frac{1}{\sigma}\right) \mathrm{e}\left(\frac{m p-n \bar{p}}{q}\right)
$$

This is hence equal to

$$
\sum_{m, n \in \mathbb{Z}} \hat{f}_{m, n}\left(\frac{1}{\sigma}\right) \frac{K(m,-n ; q)}{\varphi(q)}
$$

where $K(m,-n ; q)$ is Kloosterman sum. The formula (32) then reads,

$$
\frac{1}{\varphi(q)} \sum_{p \in \mathbb{Z}_{q}^{\times}} f\left(\frac{p}{q}, \frac{p}{q}+\mathrm{i} \frac{\sigma}{q^{2}}\right)=\hat{f}_{0,0}\left(\frac{1}{\sigma}\right)+\sum_{(m, n) \neq(0,0)} \hat{f}_{m, n}\left(\frac{1}{\sigma}\right) \frac{K(m,-n ; q)}{\varphi(q)} .
$$

Hence,

$$
\begin{aligned}
\left|\frac{1}{\varphi(q)} \sum_{p \in \mathbb{Z}_{q}^{\times}} f\left(\frac{p}{q}, \frac{p}{q}+\mathrm{i} \frac{\sigma}{q^{2}}\right)-\hat{f}_{0,0}\left(\frac{1}{\sigma}\right)\right| & \leq \sum_{(m, n) \neq(0,0)}\left|\hat{f}_{m, n}\left(\frac{1}{\sigma}\right) \frac{K(m,-n ; q)}{\varphi(q)}\right| \\
& \leq \sum_{(m, n) \neq(0,0)}\left|\hat{f}_{m, n}\left(\frac{1}{\sigma}\right)\right| C_{\varepsilon} q^{-\frac{1}{2}+\varepsilon} \underbrace{\operatorname{gcd}(|m|,|n|, q)^{\frac{1}{2}}}_{\leq \max \{|m|,|n|\}^{1 / 2}} \\
& \leq C_{\varepsilon} K_{\sigma}(f) q^{-\frac{1}{2}+\varepsilon} .
\end{aligned}
$$

The second inequality follows from the Weil's classic bound on Kloosterman sums, see Lemma 1.

\section{Proof of Theorem 2}

The Iwasawa decomposition gives a convenient parametrization of $G$. It is defined as follows: For given $g=\left(\begin{array}{ll}a & b \\ c & d\end{array}\right) \in G$ there is a unique $x, y, \phi$ such that

$$
\left(\begin{array}{ll}
a & b \\
c & d
\end{array}\right)=\left(\begin{array}{ll}
1 & x \\
0 & 1
\end{array}\right)\left(\begin{array}{cc}
y^{1 / 2} & 0 \\
0 & y^{-1 / 2}
\end{array}\right)\left(\begin{array}{cc}
\cos (\phi) & \sin (\phi) \\
-\sin (\phi) & \cos (\phi)
\end{array}\right)
$$

with $x \in \mathbb{R}, y \in \mathbb{R}_{>0}$ and $\phi \in[0,2 \pi)$.

The curve $\{(x+i y, 0) \mid x \in \mathbb{R}\}$ is an example of an orbit of the horocyclic flow on $T^{1} \mathbb{H}$. $\Gamma$ is non-compact as it has a cusp at infinity, and that curve will then be closed in the unit tangent bundle $T^{1} \mathscr{M}_{1}$, with length $\frac{1}{y}$.

Proof. For given $p, q$, we have

$$
\left(\begin{array}{ll}
a & b \\
c & d
\end{array}\right)\left(\frac{p}{q}+\mathrm{i} \frac{\sigma}{q^{2}}, 0\right)=\left(\frac{a\left(\frac{p}{q}+\mathrm{i} \frac{\sigma}{q^{2}}\right)+b}{c\left(\frac{p}{q}+\mathrm{i} \frac{\sigma}{q^{2}}\right)+d}, \phi_{\gamma}\right) .
$$

By substituting $c=q$ and $d=-p$, and $\phi=0$ we clearly have the angle

$$
\phi_{\gamma}=\phi+\arg (c z+d)=\arg (c z+d)=\arg \left(q\left(\frac{p}{q}+\mathrm{i} \frac{\sigma}{q^{2}}\right)-p\right)=\arg \left(\mathrm{i} \frac{\sigma}{q}\right)=\frac{\pi}{2} .
$$

We therefore get

$$
\left(\begin{array}{ll}
a & b \\
c & d
\end{array}\right)\left(\frac{p}{q}+\mathrm{i} \frac{\sigma}{q^{2}}, 0\right)=\left(-\frac{\bar{p}}{q}+\mathrm{i} \frac{1}{\sigma}, \frac{\pi}{2}\right)
$$


Since $f$ is invariant under $\Gamma$, then it reads

$$
\frac{1}{\varphi(q)} \sum_{p \in \mathbb{Z}_{q}^{\times}} f\left(\frac{p}{q}, \frac{p}{q}+\mathrm{i} \frac{\sigma}{q^{2}}, 0\right)=\frac{1}{\varphi(q)} \sum_{p \in \mathbb{Z}_{q}^{\times}} f\left(\frac{p}{q},-\frac{\bar{p}}{q}+\mathrm{i} \frac{1}{\sigma}, \frac{\pi}{2}\right)
$$

We apply Fourier series expansion as we did in the proof of Theorem 1,

$$
f(\xi, z, \phi)=\sum_{m, n \in \mathbb{Z}} \hat{f}_{m, n}(y, \phi) e(m \xi+n x)
$$

where $\hat{f}_{m, n}(y, \phi)=\int_{0}^{1} \int_{0}^{1} f(\xi, x+\mathrm{i} y, \phi) e(-m \xi-n x) d \xi d x$ with $y=\frac{1}{\sigma}$ and $\phi=\frac{\pi}{2}$. We hence have

$$
\frac{1}{\varphi(q)} \sum_{p \in \mathbb{Z}_{q}^{\times}} \sum_{m, n \in \mathbb{Z}} \hat{f}_{m, n}\left(\frac{1}{\sigma}, \frac{\pi}{2}\right) e\left(\frac{m p-n \bar{p}}{q}\right)=\int_{0}^{1} \int_{0}^{1} f\left(\xi, x+\mathrm{i} \frac{1}{\sigma}, \frac{\pi}{2}\right) d \xi d x+\sum_{m, n \in \mathbb{Z}} \hat{f}_{m, n}\left(\frac{1}{\sigma}, \frac{\pi}{2}\right) \frac{K(m,-n ; q)}{\varphi(q)}
$$

By Lemma 1,

$$
\begin{aligned}
\left|\frac{1}{\varphi(q)} \sum_{p \in \mathbb{Z}_{q}^{\times}} f\left(\frac{p}{q}, \frac{p}{q}+\mathrm{i} \frac{\sigma}{q^{2}}, 0\right)-\int_{\mathbb{T}^{2}} f\left(\xi, x+\mathrm{i} \frac{1}{\sigma}, \frac{\pi}{2}\right) d \xi d x\right| & \leq \sum_{m, n \in \mathbb{Z}}\left|\hat{f}_{m, n}\left(\frac{1}{\sigma}, \frac{\pi}{2}\right) \frac{K(m,-n ; q)}{\varphi(q)}\right| \\
& \leq C_{\varepsilon} N_{\sigma}(f) q^{-1 / 2+\varepsilon}
\end{aligned}
$$

the proof is then completed.

\section{Competing interests}

The authors declare that they have no competing interests.

\section{Authors' contributions}

All authors have contributed to all parts of the article. All authors read and approved the final manuscript.

\section{References}

[1] B. Bekka and M. Mayer, Ergodic theory and topological dynamics of group actions on homogeneous spaces. LMS lecture note series 269(4), Cambridge University Press, 2000, 839-853.

[2] E. Demirci Akarsu, Short incomplete Gauss sums and rational points on metaplectic horocycles, Int J Number Thr. 10(6), 2014, $1553-1576$.

[3] M. Einsiedler, S. Mozes, N. Shah and U. Shapira, Equidistribution of primitive rational points on expanding horospheres, Composito Math.152, 2016, 667-692.

[4] A. Eskin and C. McMullen, Mixing, counting and equidistribution in Lie groups, Duke Math. J. 71(1), 1993, 181-209.

[5] T. Esterman, On Kloosterman's sum, Mathematika. 8, 1961, 83-86.

[6] G. H. Hardy and E. M. Wright, An introduction of theory of numbers, Oxford Science Publications. 2003.

[7] D. A. Hejhal, On the uniform equidistribution of long closed horocycles, Loo-Keng Hua: a great mathematician of the twenty century. Asian J. Math. 4(4), 2000, 839-853.

[8] P. Sarnak, Asymptotic behavior of periodic orbits of the horocycle flow and Einstein series, Communications on Pure and Applied Math. 34, 1981, 719-739. 
[9] A. Strömbergsson, On the uniform equidistribution of long closed horocycles, Duke Mat. J. 123(3),2004, 507-547.

[10] D. Zagier, Eisenstein series and Riemann zeta functions. in: Automorphic Forms, Representation Theory and Arithmetic (Bombay 1979), Tata Inst. Fund. Res. Studies in Math. 10, 1981, 275-301. 